\title{
Estimation of Chemical Reaction Kinetics Using Ultrasound
}

\author{
Johan E. Carlson \\ Div. of Systems and Interaction \\ Dept. of Computer Science and Electrical Engineering \\ Luleå University of Technology \\ SE-971 87 Luleå, Sweden \\ Email: Johan.Carlson@1tu.se
}

\author{
Veli-Matti Taavitsainen \\ Dept. of Mathematics \\ EVTEK University of Applied Sciences \\ FI-02650 Espoo \\ Finland \\ Email: Veli-Matti.Taavitsainen@wise.evtek.fi
}

\begin{abstract}
In many ultrasound measurement situations, deriving models for the acoustic wave propagation through the system being studied is complicated. In such cases, we are often limited to study correlations between observed acoustic properties and the underlying physical properties. Sometimes this can be automated by use of statistical or empirical models. However, this often requires extensive calibration, and it does not provide as much understanding of the underlying system as we would like.

In this paper we present a general methodology for estimation of parameters of physical models based on indirect observations. The principle is demonstrated for a system where the kinetic behavior of a chemical reaction is modeled, and where measurements of ultrasound attenuation are used to estimate the model parameters. Experimental results show that we can use ultrasound to measure mass fractions of the different constituents as a function of the reaction time.

Index Terms-Implicit calibration, ultrasound measurements, physical modeling, reaction kinetics, bone cement.
\end{abstract}

\section{INTRODUCTION}

The purpose of any measurement system is to obtain information and understanding of the system being studied. It could be for example a system for process diagnostics or for material characterization. Regardless of the application at hand, we often start by developing a model of some dynamics of the process. A common approach is to develop a model of something we can observe, as a function of what we would like to know. For example, in ultrasonic measurement system, we could try to model the wave propagation through the system as a function of some mechanical properties of interest. For complex systems and system varying over time, this approach becomes infeasible, resulting in models which are either oversimplified or very complex. If the model is too simple, it might not capture all significant variations of the process. On the other hand, if the model is too complex, estimating model parameters from measured data, i.e. solving the inverse problem, becomes numerically challenging.

In some cases, this problem is avoided by resorting to purely empirical or statistical modeling, e.g. multivariate statistical analysis, neural networks, or simple linear or non-linear models. This approach helps us exploit correlations between observed data and variations in the process and as such it may be adequate. However, there are a couple of drawbacks:

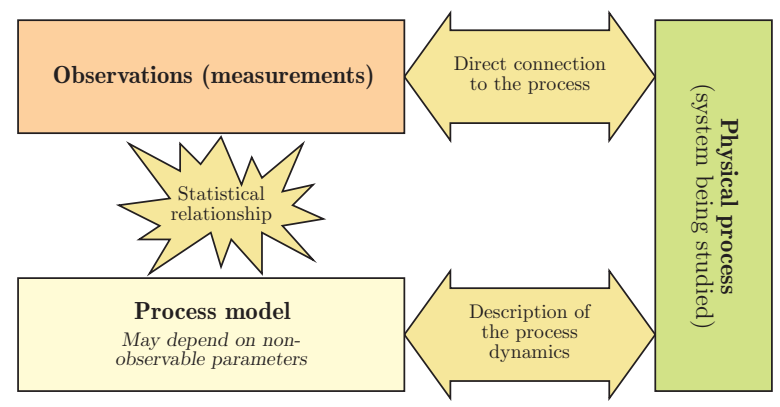

Fig. 1. Principle of model identification based on indirect observations.

- Since the model is not directly connected to any underlying physical properties of the system, we are only able to study correlations with the observed variations. In other words, the lack of causality limits the understanding of the underlying system.

- Empirical or statistical models require calibration in order to work. If the measurement conditions vary over time, we either need extensive and repeated calibration or the system will eventually break down. When they fail, it is sometimes difficult to analyze why.

In this paper we propose a different strategy, which combines the power of statistical methods with a solid physical model of the problem. As we will show, it is possible to estimate parameters of a physical model from measured data, even if the observations have no direct link to the model itself. For example, the evolution of a chemical reaction will change the mechanical properties of the substance, and as a consequence, observable acoustic properties will also change. We may be able to model the dynamics (or kinetics) of the chemical reaction, but we are not able to model the wave propagation as a function of this. However, the variations in acoustic properties are correlated to the variations in the chemical substance. We will show here how this correlation can be exploited in order to estimate parameters of the chemical kinetics model. Fig. 1 illustrates the idea, which can be summarized as: Model what you can, then measure something that is correlated to the variations predicted by the model. 
The principle, called implicit calibration [1], is illustrated here with an example, but the general idea can be used in many other applications. The example is on the setting reaction of injectable calcium sulfate bone cements. Injectable bone cements [2] based on calcium sulfate and calcium phosphates are potential materials for bone defect filling and reinforcement of osteoporotic bone. For both researchers and medical personnel it is of interest to know how the chemical reaction evolves over time. For researchers it helps developing the materials, and for medical personnel it provides guidelines for the clinical use of the materials. The problem of measuring the setting time has been studied previously by others [3], [4], but without including any models of the underlying chemistry.

The remainder of the paper is organized as follows: First the model of the reaction kinetics for the setting of calcium sulfate cement is presented, then the ultrasonic measurement principle is described, followed by the algorithms for estimating the model parameters and some experimental results.

\section{THEORY}

\section{A. Reaction kinetics}

Calcium sulfate hemihydrate (CSH) reacts with water, forming calcium sulfate dihydrate (CSD), according to the following reaction

$$
\mathrm{CaSO}_{4} \cdot \frac{1}{2} \mathrm{H}_{2} \mathrm{O}+\frac{3}{2} \mathrm{H}_{2} \mathrm{O} \rightarrow \mathrm{CaSO}_{4} \cdot 2 \mathrm{H}_{2} \mathrm{O} .
$$

Several reaction mechanisms have been proposed for this reaction. The most common one is the semi-empirical Avrami equation [5]. A comparative study of different models is given in Hand [5]. The reaction is assumed to have a nucleation period during which microscopic small dihydrate crystals are formed. After the nucleation period the main reaction starts and it is either diffusion controlled or surface controlled depending on the supersaturation conditions of the mixture. It is generally accepted that the apparent reaction order with respect to the mass fraction of hemihydrate depends on the controlling mechanism. According to these principles, the following kinetic mechanism is proposed [6]

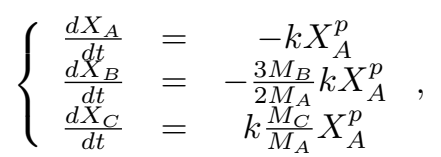

where the subscripts $A, B$, and $C$ denote $\mathrm{CSH}$, water, and CSD, respectively. The corresponding mass fractions are denoted by $X_{A}, X_{B}$ and $X_{C}$, and their molecular weights are denoted by $M_{A}, M_{B}$ and $M_{C}$. The apparent reaction order is denoted by $p$ and the reaction rate by $k$. In addition to this, the main reaction is assumed to start after the nucleation period $t_{0}$. The model contains three unknown parameters $(k, p$ and $t_{0}$ ) to be estimated from the ultrasonic measurements using implicit calibration.

\section{B. Ultrasound measurement principle}

In this work, we used the pulse-echo setup as described in Fig. 2.

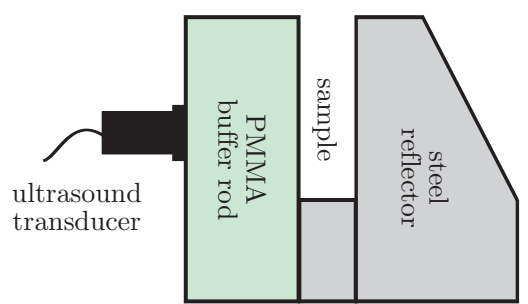

Fig. 2. The pulse-echo setup used in the measurements.

An ultrasound transducer transmits a short pulse, generally unknown. This is then reflected at the boundary between the buffer rod and the sample, and at the boundary between the sample and the back reflector. These two echoes are then recorded, and can be used to calculate the attenuation and speed of sound through the sample material. If the acoustic properties of the buffer material are known, additional properties can be calculated, such as acoustic impedance, adiabatic bulk modulus, and density [7]. In this paper we used only the spectral amplitude of the echo from the buffer rod/sample interface. Throughout the setting reaction pulses were recorded using a digitizing oscilloscope (see Sec. III-A for details). The discrete versions of the pulses are denoted $p_{k}[m]$, where the subscripts $k=1,2, \ldots, N$ correspond to different times during the setting reaction. Their spectral representations, $P_{k}[q]$ were calculated using the discrete Fourier transform, i.e.

$$
P_{k}[q]=\sum_{m=1}^{M} p_{k}[m] e^{\frac{-j 2 \pi(q-1)(m-1)}{M}},
$$

where $M$ is the length of the sampled signal. The spectral amplitudes, $y_{k}[q]$ are then calculated as

$$
y_{k}[q]=\left|P_{k}[q]\right|=\sqrt{\Re\left\{P_{k}[q]\right\}^{2}+\Im\left\{P_{k}[q]\right\}^{2}},
$$

where $\Re\{\cdot\}$ and $\Im\{\cdot\}$ denote the real and imaginary parts, respectively. These were stored in the matrix $\mathbf{Y}$, defined as

$$
\mathbf{Y}=\left[\begin{array}{llll}
\mathbf{y}_{1} & \mathbf{y}_{2} & \cdots & \mathbf{y}_{N}
\end{array}\right]^{T},
$$

where $\mathbf{y}_{k}=\left[y_{k}[1], y_{k}[2], \ldots, y_{k}[M]\right]^{T}$. In the following, the matrix $\mathbf{Y}$ is considered to be our observations, or measured data.

\section{Implicit calibration}

In implicit calibration, the kinetic parameters are estimated simultaneously with the parameters of the calibration model linking the measured ultrasonic amplitude spectra to the mass fractions of the three compounds. The implicit calibration can be made in two different ways, in the indirect and in the direct way. In the former, the measured amplitude spectra at different times are calibrated to match the modeled mass fractions using some multivariate calibration technique, e.g. PLS regression [8]. PLS regression is a good choice because it extract the part of the observations that is correlated with the mass fractions. In the latter, the modeled mass fractions are calibrated to match the measured amplitude spectra at different times, typically 
using ridge regression with non-negativity constraints. In order to clarify the method, we will use the notation that follows.

Let $\mathbf{Y}$ be the matrix of measured amplitude spectra where the $i$ 'th row contains the spectrum measured at the $i$ 'th measurement time. Let $\mathbf{X}_{\boldsymbol{\theta}}$ be the matrix of modeled mass fractions (calculated by solving Eq. (1)), where $\boldsymbol{\theta}=\left[k, p, t_{0}\right]$ is the vector of the unknown parameters and the $i$ 'th row contains the modeled mass fractions at the $i$ 'th measurement time. Now the direct implicit calibration scheme can be expressed as the following regression model

$$
\mathbf{Y}=\mathbf{X}_{\boldsymbol{\theta}} \mathbf{B}_{\boldsymbol{\theta}}+\mathbf{E}=\widehat{\mathbf{Y}}_{\boldsymbol{\theta}}+\mathbf{E},
$$

where $\mathbf{B}_{\boldsymbol{\theta}}$ represents the pure component amplitude spectra, known to be non-negative and $\mathbf{E}$ represents a matrix of residual errors.

The indirect implicit calibration scheme can be expressed as the following regression model

$$
\mathbf{X}_{\boldsymbol{\theta}}=\mathbf{Y B}_{\boldsymbol{\theta}}+\mathbf{E}=\widehat{\mathbf{X}}_{\boldsymbol{\theta}}+\mathbf{E}
$$

where $\mathbf{B}_{\boldsymbol{\theta}}$ represents the matrix of the regression coefficients and $\mathbf{E}$ represents a matrix of residual errors. Note that $\mathbf{B}_{\boldsymbol{\theta}}$ is not the same matrix in the alternative calibration schemes.

The simple idea behind implicit calibration is that the unknown matrix $\mathbf{B}_{\boldsymbol{\theta}}$ is estimated simultaneously with $\boldsymbol{\theta}$ minimizing the sum of squares norm of $\mathbf{E}$ or some other measure for the goodness of fit [1].

Because estimation of $\boldsymbol{\theta}$ is a nonlinear problem and estimation of $\mathbf{B}_{\boldsymbol{\theta}}$ is a linear problem, assuming constant $\boldsymbol{\theta}$, efficient optimization requires separation of the linear and nonlinear steps.

1) Algorithm 1 (direct implicit calibration):

1) Make an initial guess for $\boldsymbol{\theta}$.

2) Using the present value of $\boldsymbol{\theta}$, solve the system of differential equations (1) which gives the matrix $\mathbf{X}_{\boldsymbol{\theta}}$ in Eq. (5).

3) Estimate the matrix $\mathbf{B}_{\boldsymbol{\theta}}$ using ridge regression with nonnegativity constraints and calculate $\widehat{\mathbf{Y}}_{\boldsymbol{\theta}}=\mathbf{X}_{\boldsymbol{\theta}} \mathbf{B}_{\boldsymbol{\theta}}$ and $\mathbf{E}=\mathbf{Y}-\widehat{\mathbf{Y}}_{\boldsymbol{\theta}}$. Based on $E$, a value of the cost function to be minimized is then calculated.

4) Calculate a new value for $\boldsymbol{\theta}$ using the chosen optimization algorithm, e.g. the simplex algorithm or the some gradient-based method.

5) Repeat steps $2-4$ until the chosen convergence criteria are met.

2) Algorithm 2 (indirect implicit calibration):

1) Make an initial guess for $\boldsymbol{\theta}$.

2) Using the present value of $\boldsymbol{\theta}$, solve the system of differential equations (1) which gives the matrix $\mathbf{X}_{\boldsymbol{\theta}}$ in Eq. (6).

3) Estimate the matrix $\mathbf{B}_{\theta}$ (in Eq. (6)) using PLS regression and calculate $\hat{\mathbf{X}}_{\boldsymbol{\theta}}=\mathbf{Y B}_{\boldsymbol{\theta}}$ and $R^{2}$ between $\mathbf{X}$ and $\widehat{\mathbf{X}}_{\boldsymbol{\theta}}$. This is then the objective function of the optimization algorithm.
4) Calculate a new value for $\boldsymbol{\theta}$ using the chosen maximization algorithm, e.g. the simplex algorithm or the some gradient-based method.

5) Repeat steps $2-4$ until the chosen convergence criteria are met.

\section{EXPERIMENTS}

\section{A. Materials and methods}

$30 \mathrm{~g} \alpha$-calcium sulfate hemihydrate $(\mathrm{CSH})$ powder (Bo Ehrlander AB, Gothenburg, Sweden) was mixed with an aqueous solution of $2.5 \%$ (by weight of $\mathrm{Na}_{2} \mathrm{HPO}_{4}$ at a liquidto-powder (L/P) ratio of $0.32 \mathrm{~mL} \mathrm{~g}^{-1}$, during one minute to form a paste. The mixing time was 1 minute, after which the paste was considered homogeneous. Thereafter it was put inside the measurement cell (Fig. 2) and formed so that it completely covered the area of the transducer surface. The ultrasonic measurement was started 3 minutes after the initial mixing started. The data acquisition then continued for two hours, with one pulse-echo measurement every 20 seconds. All measurements were performed in a climate chamber at $37{ }^{\circ} \mathrm{C} \pm 1{ }^{\circ} \mathrm{C}$.

The pulse-echo measurements were made using a $2 \mathrm{MHz}$ ultrasound transducer from Ceram AB (Lund, Sweden). The transducer was excited using a Panametrics P5800 pulser/receiver. The received pulse-echoes were sampled at $200 \mathrm{MHz}$ using a Nicolet 460 digitizing oscilloscope with a vertical resolution of 8 bits. All further processing was done in MATLAB (The MathWorks, Inc.).

\section{B. Results}

The implicit calibrations were carried out using both the direct and indirect methods. In the indirect method, the PLS dimension and the method of scaling for the measurement data $(\mathbf{Y})$ have to be chosen. In this work, the columns of $\mathbf{Y}$ were mean centered, but otherwise not scaled. Theoretically, because the matrix $\mathbf{X}_{\boldsymbol{\theta}}$ is of rank one, due to the stoichiometry, only one PLS dimension should be needed.

First we estimated all three unknown parameters, $k, t_{0}$ and $p$ simultaneously. All optimizations were carried out using the Nelder-Mead simplex algorithm. Both methods gave an a value of the apparent reaction order, $p \approx 2.3$ suggesting, according to e.g. Hand [5] and Niemann [6], a surface controlled reaction. Since the fit was practically as good with the value $p=2$, we fixed the apparent reaction order to this value and re-estimated the other two parameters.

Fig. 3 shows the resulting mass fraction estimates from using the indirect calibration. For the direct calibration the results for the mass fractions were identical and are not plotted here. However, using the direct calibration scheme, we are also able to estimate the measure amplitude spectra. This is shown in Fig. 4, for different times during the reaction. Early in the reaction it is clear that the model is wrong (we actually assume the reaction starts after a time $t=t_{0}$, which is clearly not true). As the reaction progresses, however, we see that the estimated amplitude spectra agree well with the measurements. 

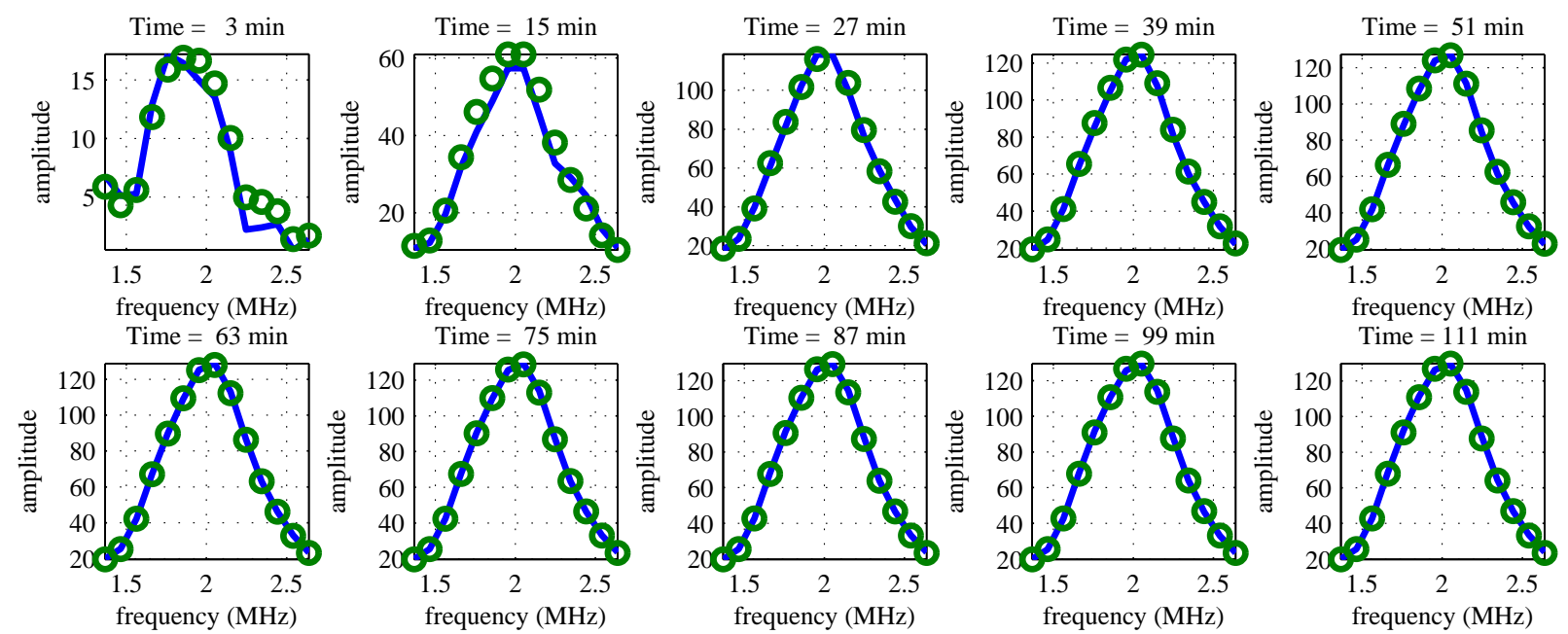

Fig. 4. Modeled (o) and measured (solid) spectral amplitudes as a function of the setting time (direct calibration).

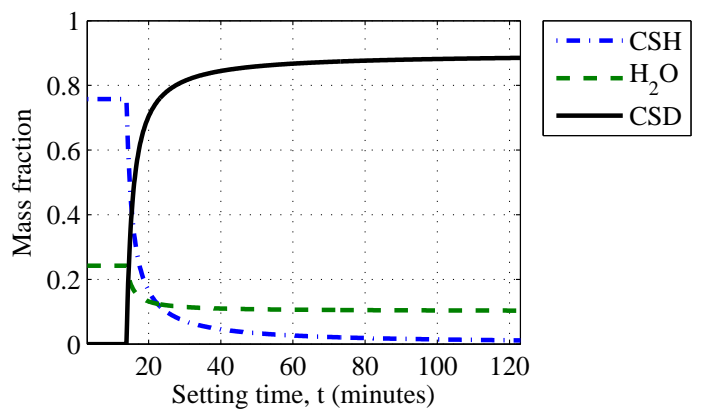

Fig. 3. Estimated mass fractions as a function of the reaction time (indirect calibration).

\section{Discussion}

The results of the mass fraction estimation of CSD (Fig. 3) show significant similarities with the results from [4]. In the previous paper, we examined only how certain acoustic properties changed during the setting reaction, and made no attempt in interpreting this with respect to any underlying mechanism. In the present work, however, we are able to draw the conclusions that the variation in acoustic impedance noticed in [4] is to a large extent due to the increased mass fraction of CSD.

In addition to the kinetics model used in this paper, we also evaluated a couple of other candidate models. However, since the purpose of this paper is on a general demonstration of principle rather than on solving the specific problem of this reaction, readers interested in more detail regarding this specific reaction are referred to [9].

\section{CONCLUSiOnS}

In this paper we explained the principle of direct and indirect implicit calibration. We then showed by an example that this strategy can be used to estimated parameters of models that are only correlated to the available observations.
In the specific example of the setting reaction of calcium sulfate cements, we showed that we can estimate the mass fractions of the different constituents of the reaction, as well as the reaction order the reaction rate. This shows that implicit calculation can be used to exploit correlations between observed acoustic properties and underlying physical properties of interest, without having to explicitly model how ultrasound propagation is affected by the system under observation.

\section{ACKNOWLEDGMENTS}

The authors wish to express their sincerest gratitude toward Dr. Malin Nilsson at BoneSupport $\mathrm{AB}$ for providing the sample material and for her valuable comments.

\section{REFERENCES}

[1] V.-M. Taavitsainen, H. Haario, and M. Laine, "Rapid estimation of kinetics by implicit calibration. II," J. Chemometrics, vol. 17, pp. 140 $150,2002$.

[2] M. Nilsson, E. Fernández, S. Sarda, L. Lidgren, and J. A. Planell, "Characterisation of a Novel Calcium Phosphate/Sulphate Bone Cement," J. Biomed. Mater. Res., vol. 61, pp. 600-607, 2002.

[3] A. M. Viano, J. A. Auwarter, J. Y. Rho, and B. K. Hoffmeister, "Ultrasonic Characterization of the Curing Process of Hydroxyapatite-Modified Bone Cement," J. Biomed Mater. Res., vol. 56, pp. 593-599, 2001.

[4] J. Carlson, M. Nilsson, E. Fernández, and J. A. Planell, "An Ultrasonic Pulse-Echo Technique for Monitoring the Setting of $\mathrm{CaSO}_{4}$-Based Bone Cement," Biomaterials, vol. 24, no. 1, pp. 71-77, 2003.

[5] R. J. Hand, "The kinetics of hydration of calcium sulphate hemihydate: a critical comparison of the models in the literature," Cement and Concrete Research, vol. 24, pp. 885-895, 1994.

[6] L. Niemann, Die Reaktionskinetik des Gipsabbindens: Makroskopische Reaktionsraten und Mechanismen in molekularem Maßstab. Universitätsverlag Karlsruhe, 2005, vol. 28.

[7] L. E. Kinsler, A. R. Frey, A. B. Coppens, and J. V. Sanders, Fundamentals of Acoustics, 3rd ed. New York: Wiley, 1982.

[8] S. Wold, M. Sjöström, and L. Eriksson, "PLS regression: A basic tool of chemometrics," Chemometrics Intell. Lab. Syst., vol. 58, pp. 109-130, 2001 .

[9] J. E. Carlson and V.-M. Taavitsainen, "Ultrasonic measurement of the reaction kinetics of the setting of calcium sulfate cements using implicit calibration," J. Chemom., vol. Accepted, in press, 2008. 